\title{
Morphine Sulfate Sustained-Release Tablet
}

National Cancer Institute

\section{Source}

National Cancer Institute. Morphine Sulfate Sustained-Release Tablet. NCI Thesaurus. Code $C 84853$.

A sustained-release tablet formulation containing the sulfate salt of the opiate alkaloid morphine with analgesic activity. Morphine binds to and activates the mu-opioid receptors in the central nervous system (CNS), thereby mimicking the effects of the endogenous opioids. Binding of morphine to opioid receptors stimulates exchange of GTP for GDP, inhibits adenylate cyclase, and decreases intracellular cAMP. This inhibits the release of various nociceptive neurotransmitters, such as substance $P$, gammaaminobutyric acid (GABA), dopamine, acetylcholine, noradrenaline, vasopressin, and somatostatin. In addition, morphine closes N-type voltage-gated calcium channels and opens calcium-dependent inwardly rectifying potassium channels, which results in hyperpolarization of neuronal membranes and a reduction in neuronal excitability, and subsequently, analgesia and sedation. 